Check for updates

Cite this: Phys. Chem. Chem. Phys., 2017, 19, 19756

Received 7th April 2017

Accepted 9th June 2017

DOI: $10.1039 / c 7 c p 02258 a$

rsc.li/pccp

\title{
2-Thiouracil intersystem crossing photodynamics studied by wavelength-dependent photoelectron and transient absorption spectroscopies $\dagger$
}

\author{
José A. Sánchez-Rodríguez, $\ddagger^{a}$ Abed Mohamadzade, $\ddagger^{a}$ Sebastian Mai, (D) $\ddagger^{b}$ \\ Brennan Ashwood, (D) $\ddagger^{\mathrm{C}}$ Marvin Pollum, ${ }^{\mathrm{C}}$ Philipp Marquetand, (DD \\ Leticia González, (D) *b Carlos E. Crespo-Hernández (D) *c and Susanne Ullrich*a
}

\begin{abstract}
Single-atom substitution within a natural nucleobase-such as replacing oxygen by sulfur in uracil-can result in drastic changes in the relaxation dynamics after UV excitation. While the photodynamics of natural nucleobases like uracil are dominated by pathways along singlet excited states, the photodynamics of thiobases like 2-thiouracil populate the triplet manifold with near unity quantum yield. In the present study, a synergistic approach based on time-resolved photoelectron spectroscopy (TRPES), time-resolved absorption spectroscopy (TRAS), and ab initio computations has been particularly successful at unraveling the underlying photophysical principles and describing the dissimilarities between the natural and substituted nucleobases. Specifically, we find that varying the excitation wavelength leads to differences between gas-phase and condensed-phase experimental results. Systematic trends are observed in the intersystem crossing time constants with varying excitation wavelength, which can be readily interpreted in the context of $a b$ initio calculations performed both in vacuum and including solvent effects. Thus, the combination of TRPES and TRAS experiments with highlevel computational techniques allows us to characterize the topology of the potential energy surfaces defining the relaxation dynamics of 2-thiouracil in both gas and condensed phases, as well as investigate the accessibility of conical intersections and crossings, and potential energy barriers along the associated relaxation coordinates
\end{abstract}

\section{Introduction}

Canonical nucleobases found in DNA and RNA possess inherent photoprotective mechanisms against harmful UV radiation, featuring primarily ultrafast excited-state relaxation to the ground state, either directly or through intermediate singlet excited states. $^{1-11}$ Thiobases-structurally modified nucleobases where a sulfur atom substitutes the oxygen atom in a carbonyl group-have revealed how single-atom substitution can drastically affect the accessibility of these relaxation pathways. ${ }^{7,12-21}$ Molecules such as 6-thioguanine $e^{17,22,23}$ 4-thiothymine, ${ }^{13,14,16,18,24}$

\footnotetext{
${ }^{a}$ Department of Physics and Astronomy, University of Georgia, Athens, GA 30602, USA.E-mail: ullrich@physast.uga.edu

${ }^{b}$ Institute of Theoretical Chemistry, Faculty of Chemistry, University of Vienna, Währinger Straße 17, 1090 Vienna, Austria. E-mail: leticia.gonzalez@univie.ac.at ${ }^{c}$ Department of Chemistry and Center for Chemical Dynamics, Case Western Reserve University, 10900 Euclid Avenue, Cleveland, Ohio 44106, USA.

E-mail: carlos.crespo@case.edu

$\dagger$ Electronic supplementary information (ESI) available. See DOI: 10.1039/ c7cp02258a

\$ These authors contributed equally to this work.
}

and 2-thiocytosine ${ }^{21}$ exhibit femtosecond intersystem crossing (ISC) dynamics that result in nearly unity triplet yields. The population of the triplet state prepares a highly reactive molecule, which by itself, or through diffusional encounter with molecular oxygen to generate singlet oxygen, ${ }^{18,22,23}$ can damage biomolecules within the cell. ${ }^{25-27}$ Hence, not only how the triplet states are populated in these thiobases, but also the magnitude of their triplet-state lifetimes, are of special interest in phototherapeutic applications. ${ }^{18,20,28-30}$

Besides the 6-thioguanine, 4-thiothymine, and 2-thiocytosine monomers, other thiobases such as 2-thiouracil also populate the reactive triplet state in high yields and on an ultrafast time scale. However, the dynamics responsible for ISC to the triplet state and then again back to the ground state are not fully elucidated, and are the subject of the present study. Early experiments with 2 -thiouracil in solution ${ }^{19,31}$ provided evidence for the involvement of the ${ }^{1} n \pi^{*}$ state in the ultrafast population of the ${ }^{3} \pi \pi^{*}$ state, and a triplet decay lifetime of $70 \mathrm{~ns}$ in acetonitrile was measured by Vendrell-Criado et al. ${ }^{32}$ Recently, $\mathrm{Yu}$ et $a .^{33}$ reported the first investigation of the excited-state dynamics of 2-thiouracil in the gas phase using time-resolved 
photoionization. The results revealed an ultrafast $(<100 \mathrm{fs})$ lifetime, which was assigned to internal conversion from the ${ }^{1} \pi \pi^{*}$ state to the ${ }^{1} \mathrm{n} \pi^{*}$ state, a second lifetime of $775 \mathrm{fs}$ associated with the population of the triplet manifold, and a third lifetime of $203 \mathrm{ps}$, which was assigned to the decay of the ${ }^{3} \pi \pi^{*}$ state back to the ground state. The repopulation of the ground state in few hundreds of picoseconds in the gas phase was proposed to be associated with a small energy barrier ( $c a .0 .2 \mathrm{eV})$ that needs to be overcome in order to access the triplet-singlet intersection seam and a large spin-orbit coupling of $130 \mathrm{~cm}^{-1}$ at this intersection. $^{33}$

A number of computational studies have also investigated the molecular mechanisms responsible for the efficient ISC of 2-thiouracil. The first study, by Cui and Fang, ${ }^{34}$ who employed the CASPT2//CASSCF method (computing energies with CASPT2 based on geometries obtained with CASSCF), reported three possible pathways from the initially excited $\mathrm{S}_{2}\left({ }^{1} \pi \pi^{*}\right)$ state to the lowest triplet state $\mathrm{T}_{1}\left({ }^{3} \pi \pi^{*}\right)$. The proposed pathways are $\mathrm{S}_{2}\left({ }^{1} \pi \pi^{*}\right) \rightarrow \mathrm{S}_{1}\left({ }^{1} \mathrm{n} \pi^{*}\right) \rightarrow \mathrm{T}_{1}\left({ }^{3} \pi \pi^{*}\right)$ (path I), $\mathrm{S}_{2}\left({ }^{1} \pi \pi^{*}\right) \rightarrow$ $\mathrm{T}_{2}\left({ }^{3} \mathrm{n} \pi^{*}\right) \rightarrow \mathrm{T}_{1}\left({ }^{3} \pi \pi^{*}\right)$ (path II), and $\mathrm{S}_{2}\left({ }^{1} \pi \pi^{*}\right) \rightarrow \mathrm{T}_{3}\left({ }^{3} \mathrm{n} \pi^{*}\right) \rightarrow$ $\mathrm{T}_{2}\left({ }^{3} \mathrm{n} \pi^{*}\right) \rightarrow \mathrm{T}_{1}\left({ }^{3} \pi \pi^{*}\right)$ (path III), as sketched in Fig. 1. In their computations, Cui and Fang $^{34}$ imposed planarity of the 2-thiouracil molecule in the optimization of the excited-state stationary points. This motivated less-restrictive follow-up calculations by Gobbo and Borin, ${ }^{35}$ who identified paths I and II as probable relaxation pathways. More recently, a study by some of us $^{36}$ highlighted that most of the stationary and intersection points of 2-thiouracil exhibit significant out-of-plane distortions contrary to the presumptions of Cui and Fang. ${ }^{34}$ Hence, Mai et $a .^{36}$ concluded that while the general relaxation pathways of Cui and Fang (paths I to III) correctly involve the relevant excited states, the details of the relaxation pathways (i.e., energies, energy gaps, barriers, involvement of specific structures) are

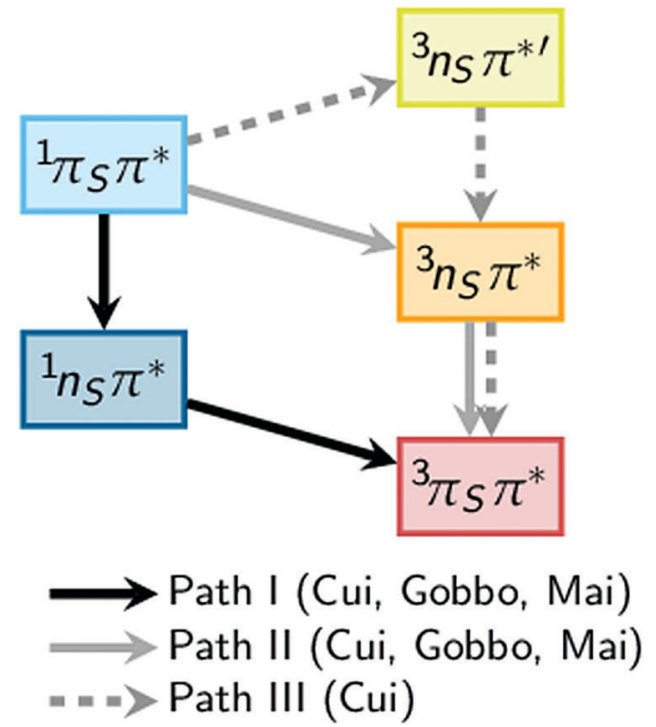

Fig. 1 Comparison of theoretical pathways for the population of the excited triplet state as calculated by Cui and Fang, ${ }^{34}$ Gobbo and Borin, ${ }^{35}$ and Mai et al. ${ }^{36}$ not reliable. Using MS-CASPT2 for both energies and geometries, ${ }^{36}$ the authors showed that $\mathrm{S}_{2} \rightarrow \mathrm{S}_{1} \rightarrow \mathrm{T}_{2} \rightarrow \mathrm{T}_{1}$ is in fact the primary relaxation pathway. Noteworthy, they also clarified $^{36,37}$ that this pathway corresponds to ${ }^{1} \pi \pi^{*} \rightarrow$ ${ }^{1} \mathrm{n} \pi^{*} \rightarrow{ }^{3} \pi \pi^{*}$ (as in path I), as the ${ }^{3} \pi \pi^{*}$ state is the secondlowest triplet state (the $\mathrm{T}_{2}$ ) at the singlet-triplet crossing after temporarily switching order with the ${ }^{3} n \pi^{*}$ state. That study also showed that this crossing seam between the ${ }^{1} n \pi^{*}$ and ${ }^{3} \pi \pi^{*}$ states is easily accessible, and that the large spin-orbit couplings (SOCs) of up to $150 \mathrm{~cm}^{-1}$ can induce population transfer to the triplet state. The magnitude of the SOCs can be explained by the change of orbital type in the ${ }^{1} n \pi^{*} \rightarrow{ }^{3} \pi \pi^{*}$ transition (following the El-Sayed rule $^{38}$ ) and the heavy-atom effect of the sulfur atom. Furthermore, the particular topology of the excited-state potentials disfavors direct relaxation to the ground state, explaining the near-unity ISC yield of 2-thiouracil. ${ }^{19}$ These findings, derived from static calculations, were more recently confirmed by MS-CASPT2-based dynamics simulations, ${ }^{37}$ which showed trajectories relaxing primarily along path I, although path II was also observed. According to the kinetic model of ref. 37 , the ${ }^{1} \pi \pi^{*} \rightarrow{ }^{1} \mathrm{n} \pi^{*}$ step occurs within less than 100 fs $\left(\tau_{1}=59 \pm 12 \mathrm{fs}\right.$ ), followed by the ${ }^{1} \mathrm{n} \pi^{*} \rightarrow{ }^{3} \pi \pi^{*}$ ISC step with a lifetime $\left(\tau_{2}\right)$ of approximately $400 \mathrm{fs}$. It was also shown that the process of relaxation towards the $S_{1}\left({ }^{1} n \pi^{*}\right)$ minimum is accompanied by strong out-of-plane motion of the sulfur atom. ${ }^{37}$

In the present work, we show interesting changes of the above-mentioned lifetimes when varying the excitation wavelength in our gas-phase (time-resolved photoelectron spectroscopy, TRPES) and condensed-phase (time-resolved absorption spectroscopy, TRAS) measurements. In particular, we find a strikingly different behavior between dynamics in the gas phase and solution. Based on ab initio simulations, we explain these findings with the presence of energy barriers to access conical intersections and singlet-triplet crossing points.

\section{Experimental details}

\section{Time-resolved photoelectron spectroscopy setup}

The apparatus used in the gas-phase experiments, including a femtosecond laser system, a gaseous molecular beam source, and a magnetic bottle photoelectron spectrometer, have been described in detail in ref. 39-43.

In order to transfer 2-thiouracil (Sigma Aldrich, $\geq 98 \%$ ) into the gas phase, the sample was placed in a quartz holder and heated to $210{ }^{\circ} \mathrm{C}$. The continuous gas molecular beam was transported to the source vacuum chamber with a helium carrier gas while being doubly skimmed. In the ionization region of the spectrometer the molecular beam and the UV femtosecond pump pulse and time-delayed probe pulse intersect, with the pump and probe beams being focused by two separate $50 \mathrm{~cm}$ lenses to spatially overlap at a small angle.

All pump pulses (249, 259.9, 271, 283 and $291.5 \mathrm{~nm}$ ) were generated using a Coherent Inc. OPerA optical parametric amplifier (OPA). The probe pulse of $329 \mathrm{~nm}$ was produced from a Coherent Inc. traveling-wave OPA (TOPAS-C). The probe 
beam wavelength was selected due to its low absorbance in 2-thiouracil, ${ }^{44}$ thus reducing the possibility of obtaining undesirable probe-pump signal. In order to promote a $1+2^{\prime}$ pump-probe scheme for ionization, the pump pulse energy was kept at 1.5-1.7 $\mu \mathrm{J}$ per pulse and the probe pulse energy at 11-15 $\mu \mathrm{J}$ per pulse.

A 50:50 mixture of 1,3-butadiene and helium was used to conduct the timing calibrations. The Gaussian cross-correlation function showed a full width at half maximum of around $200 \mathrm{fs}$, and defined the position of zero pump-probe delay. The photoelectron energy spectra were calibrated using the known ionization potentials and vibrational features of 1,3-butadiene. ${ }^{45}$

For each excitation wavelength, short-range 2D TRPES data were recorded with equal step size of 25 fs for a pump-probe delay up to $4 \mathrm{ps}$. The contributions that make up the overall TRPES signal were deconvoluted with a global analysis program written in Labview based on simultaneous fitting of the spectra and associated decay dynamics. The algorithm uses standard sequential rate equations with numerical convolution. Three individual contributions were necessary to accurately fit the measured data, such that the residual signal was negligible and random (see the $\mathrm{ESI}^{\dagger}$ in Fig. S1 for the systematic fitting residuals obtained with only two constants). Time traces of individual contributions are generated by integration over all electron energies. The dynamics of the long-lived (tens to hundreds ps) contribution were characterized in a separate long-range TRPES scan with unequal step size that extends beyond 200 ps pump-probe delay. Using a sequential triple exponential decay function convoluted with a Gaussian instrument response function, a time constant for the long (ps) decay was extracted in OriginPro from a global fit to the energyintegrated short- and long-range scans and used in the $2 \mathrm{D}$ fits described above.

\section{Femtosecond-to-microsecond time-resolved absorption setup}

TRAS was used to study the decay of the triplet state of 2-thiouracil in acetonitrile. The laser and spectrometer setup was described in significant detail in previous publications. ${ }^{19,46}$ In brief, a Ti:Sapphire oscillator seeds a chirped pulse regenerative amplifier (Coherent Libra-HE). The amplifier generates $4 \mathrm{~mJ}, 100 \mathrm{fs}$ pulses at $800 \mathrm{~nm}$ with a $1 \mathrm{kHz}$ repetition rate, which were used to pump an optical parametric amplifier (OPA, TOPAS, Quantronix/Light Conversion). A small fraction of the fundamental beam was split off prior to the OPA and focused through a constantly-moving $2 \mathrm{~mm} \mathrm{CaF}_{2}$ crystal to generate a broadband probe pulse (320-710 nm). The spectrometer (Helios, Ultrafast Systems, LLC) employs a mechanical delay stage to adjust the time it takes the probe pulse to reach the sample. The broadband probe pulses were split into two fractions. One beam passes through the sample $(2 \mathrm{~mm}$ path length) to probe the transient signals, while the other serves to correct for changes in the white light continuum throughout the experiment. The pulses were focused into optical fibers leading to CMOS detection units. Generation of the reference signal comes via a chopper wheel, which blocks every other pump pulse, providing an alternating sequence of spectra with and without sample excitation, and thus providing $\Delta A$ transient absorption data. The OPA was tuned to excitation wavelengths of 268, 290, and $316 \mathrm{~nm}$. Excitation pulses were set to an intensity of $1 \mu \mathrm{J}$ at the sample using a neutral density optical filter in order to minimize cross-phase modulation effects as well as sample degradation.

The nanosecond transient absorption experiments make use of an electronically-triggered photonic crystal fiber for probe light generation in the spectral range from $\sim 375$ to $800 \mathrm{~nm}$ (Eos, Ultrafast Systems, LLC) with a time resolution of 400 ps and a temporal window of up to $120 \mu \mathrm{s} .{ }^{46,47}$ This light source is conveniently coupled to our femtosecond transient absorption spectrometer described above, which allows the use of identical experimental conditions, signal detection, and data collection.

For these experiments, samples were prepared in acetonitrile at a concentration of $0.6 \mathrm{mM}$, unless stated otherwise, and under $\mathrm{N}_{2}$-saturated conditions to minimize diffusional quenching of the long-lived signals by molecular oxygen which may become relevant on ns-to- $\mu$ s time scales. $\mathrm{N}_{2}$-Saturated solutions were purged for 30 min prior and blanketed with $\mathrm{N}_{2}$ for the course of the experiment. Air-saturated solutions, of ca. $0.5 \mathrm{mM}$ concentration of 2-thiouracil in acetonitrile, were used during the collection of the ultrafast dynamics at excitation wavelengths of 268 and $290 \mathrm{~nm}$. A concentration of $1.2 \mathrm{mM}$ was used for ultrafast experiments performed with excitation wavelength of $316 \mathrm{~nm}$. For all transient absorption experiments, solutions were continuously stirred using a Teflon stir bar, and samples were replaced with fresh solutions before the change in absorbance reached 5\% to avoid any contamination of the transient signals by the excitation of possible photoproducts.

A homemade software (Labview, National Instruments, Inc.) was used for data collection. Approximately 30 kinetic traces in the probe spectral range from $c a$. 630 to $710 \mathrm{~nm}$ were averaged from the transient absorption band of the triplet state and fitted to a single-exponential decay function, convoluted by the instrument response function of 400 ps using Igor Pro 6.36. ${ }^{19,46}$

\section{Computational details}

In order to rationalize the observed rates for ISC to the ground state, we performed quantum chemical calculations on the MS-CASPT2(12,9)/cc-pVDZ (multi-state complete active space second-order perturbation theory) level of theory. ${ }^{48,49}$ We optimized two $T_{1}$ minima and two $T_{1} / S_{0}$ minimum-energy crossing points $^{50}$ of 2 -thiouracil, using numerical gradients and the polarizable continuum model (PCM) ${ }^{51}$ to account for the acetonitrile solvent. Because $\mathrm{T}_{1} \rightarrow \mathrm{S}_{0}$ ISC is a slow process, the PCM charges were computed self-consistently with the $\mathrm{T}_{1}$ state. The energy profiles shown below were obtained with single-point calculations on geometries obtained by linear interpolation in internal coordinates. All computations were performed with MOLCAS 8.0, ${ }^{52}$ using no IPEA shift ${ }^{61}$ and an imaginary level shift of 0.2 a.u. The optimized coordinates are given in the ESI. $\dagger$

\section{Results and discussion}

Fig. 2 presents the UV absorption spectrum of 2-thiouracil in the gas phase ${ }^{44}$ (green) and in acetonitrile solution ${ }^{19,31}$ (blue). 

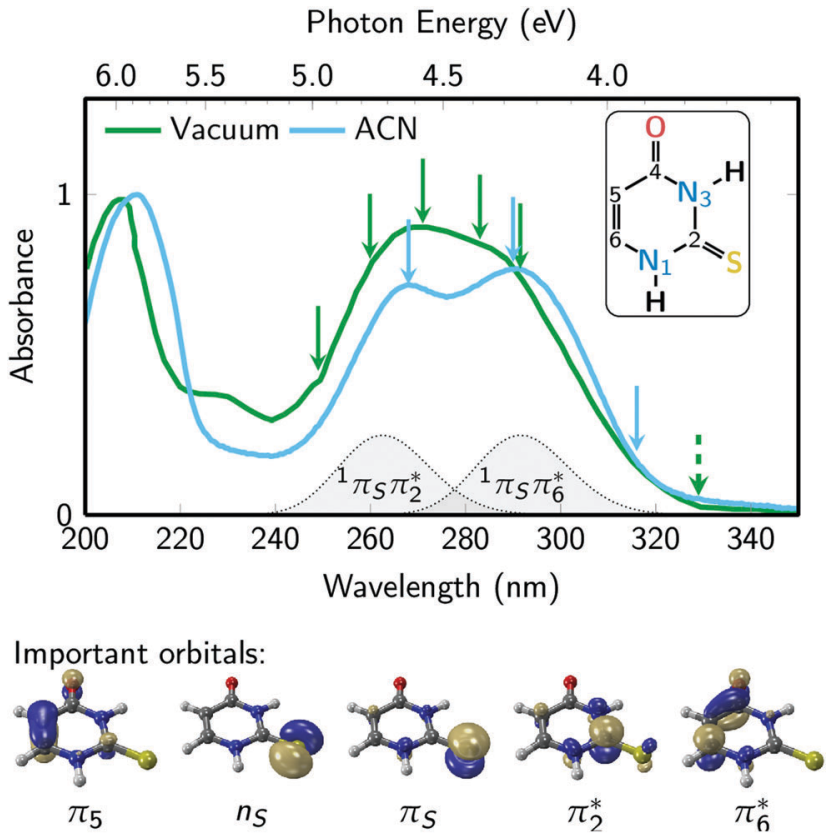

Fig. 2 2-Thiouracil absorption spectrum ${ }^{44}$ in the gas phase (green) and acetonitrile (ACN, blue) with an absorption band centered around $275 \mathrm{~nm}$ wavelength, which is due to the bright $\mathrm{S}_{2}\left({ }^{1} \pi_{\mathrm{S}} \pi_{6}{ }^{*}\right)$ and $\mathrm{S}_{4}\left({ }^{1} \pi_{\mathrm{S}} \pi_{2}{ }^{*}\right)$ states. The green arrows represent the pump wavelengths centered at 249, 259.9, 271, 283 and $291.5 \mathrm{~nm}$ and the green dashed arrow represents the $329 \mathrm{~nm}$ probe wavelength used in the TRPES experiments. The blue arrows represent the pump wavelengths $(268,290$, and $316 \mathrm{~nm})$ used in the TRAS experiments, which monitored the dynamics in the 320 to $700 \mathrm{~nm}$ probe range. The inset shows the molecular structure of 2-thiouracil's most stable tautomer, ${ }^{53,54}$ with the atomic numbering also shown. Important frontier orbitals of 2-thiouracil are shown below the spectra. These five orbitals are involved in all relevant excited states (labelled as ${ }^{1} n_{\mathrm{S}} \pi_{2}{ }^{*},{ }^{1} \pi_{\mathrm{S}} \pi_{2}{ }^{*}$, ${ }^{1} \pi_{S} \pi_{6}{ }^{*},{ }^{3} n_{S} \pi_{2}{ }^{*},{ }^{3} \pi_{S} \pi_{2}{ }^{*},{ }^{3} \pi_{5} \pi_{6}{ }^{*}$.

The green arrows mark the pump and probe wavelengths employed in the TRPES measurements, whereas the blue arrows indicate the pump wavelengths used in the TRAS experiments. Excitation occurs primarily to the lowest-energy absorption band, which ab initio calculations ${ }^{36}$ assign to the bright ${ }^{1} \pi_{\mathrm{S}} \pi_{6}{ }^{*}$ state and, towards the high-energy side of the band, the ${ }^{1} \pi_{\mathrm{S}} \pi_{2}{ }^{*}$ state (these are the $\mathrm{S}_{2}$ and $\mathrm{S}_{4}$ states at the Franck-Condon (FC) point); the energetic positions of these states are indicated in the figure. Note that from here on we adopt the state nomenclature from ref. 36 in order to distinguish between the several $n \pi^{*}$ and $\pi \pi^{*}$ states of 2-thiouracil. Accordingly, the bright ${ }^{1} \pi_{\mathrm{S}} \pi_{6}{ }^{*}$ and ${ }^{1} \pi_{\mathrm{S}} \pi_{2}{ }^{*}$ states correspond to electron promotion from the $\pi$ orbital localized at the sulfur atom into two different ring $\pi^{*}$ orbitals: $\pi_{6}{ }^{*}$ localized significantly at carbon $\mathrm{C}_{6}$ (i.e., the $\pi^{*}$ orbital of the $\mathrm{C}=\mathrm{C}$ double bond) and $\pi_{2}{ }^{*}$ at $\mathrm{C}_{2}\left(\pi^{*}\right.$ orbital of the $\mathrm{C}=\mathrm{S}$ double bond), as shown in Fig. 2 (bottom). ${ }^{36}$ The weak absorption toward longer wavelengths is associated with the ${ }^{1} n_{S} \pi_{2}{ }^{*}$ state $\left(S_{1}\right)$, a transition with small oscillator strength originating from the non-bonding sulfur orbital.

\section{Role of the excitation wavelength in the excited-state dynamics in gas phase}

The 2-thiouracil TRPES results shown in Fig. 3 are arranged from top to bottom in order of increasing excitation wavelengths with the corresponding data (column 1) and fit results presented along each row (total 2D fit in column 2; individual 2D contributions in columns 3-5; energy-integrated time traces in columns 6 and 7). All color maps are plotted as a function of electron binding energy versus pump-probe delay. The electron binding energies are calculated by subtracting the measured photoelectron kinetic energies from the total photon energy of the $1+2^{\prime}$ multiphoton excitation-ionization process.

Analysis of the photoelectron spectra relies on ionization correlations between the neutral excited and ionic states. The basic procedure has been described in detail in ref. 33 for the TRPES measurement at $291.5 \mathrm{~nm}$ excitation, which is reproduced here in the bottom row of Fig. 3. A brief summary of essential information is given below. Photoionization from all relevant excited states $\left({ }^{1} n_{\mathrm{S}} \pi_{2}{ }^{*},{ }^{1} \pi_{\mathrm{S}} \pi_{2}{ }^{*},{ }^{1} \pi_{\mathrm{S}} \pi_{6}{ }^{*},{ }^{3} \mathrm{n}_{\mathrm{S}} \pi_{2}{ }^{*},{ }^{3} \pi_{\mathrm{s}} \pi_{2}{ }^{*}\right.$, ${ }^{3} \pi_{5} \pi_{6}{ }^{*}$ ) will preferentially occur into the lowest ionization band (IP1) ${ }^{55}$ which is due to two close-lying ionic states with the hole in either the sulfur $\mathrm{n}$ or $\pi$ orbital. ${ }^{56}$ The change in ionization energy along the relaxation path was previously characterized by $a b$ initio calculations. ${ }^{33}$ Taking the destabilization of the ionic states and the stabilization of the neutral states (i.e., vibrational energy gain) during relaxation into account, photoelectron signals are expected at the total electron binding energies indicated as solid white lines in Fig. 3. For $291.5 \mathrm{~nm}$ excitation, these correspond to $8.9 \mathrm{eV}$ for ionization from the FC region, $9.7 \mathrm{eV}$ and $10.7 \mathrm{eV}$ from the ${ }^{1} \pi_{\mathrm{S}} \pi_{6}{ }^{*}$ and ${ }^{1} \pi_{\mathrm{S}} \pi_{2}{ }^{*}$ minima, respectively, and between $10.9 \mathrm{eV}$ and $10.1 \mathrm{eV}$ for ionization from two different minima $\left({ }^{3} \pi_{\mathrm{S}} \pi_{2}{ }^{*},{ }^{3} \pi_{5} \pi_{6}{ }^{*}\right)$ in the triplet manifold. This information provides the basis for identification of the electronic excited states participating in the three-step relaxation process. Column 3 displays the shortlived TRPES signal following excitation to the bright ${ }^{1} \pi_{\mathrm{S}} \pi^{*}$ states and the subsequent relaxation towards the minimum of the ${ }^{1} \pi_{\mathrm{S}} \pi_{6}$ state. The observed broad photoelectron band from ionization into IP1 (FC) and IP1 $\left({ }^{1} \pi_{\mathrm{S}} \pi_{6}{ }^{*} \mathrm{~min}\right)$ confirms this assignment. An approximately $1 \mathrm{eV}$ shift of the photoelectron band towards higher electron binding energies in column 4 is in agreement with the calculated increase in IP1 for ionization from the ${ }^{1} n_{S} \pi_{2}{ }^{*}$ minimum (IP1 $\left({ }^{1} n_{S} \pi_{2}{ }^{*}\right.$ min)) plus vibrational energy gain, and the TRPES signal is therefore assigned to the ${ }^{1} n_{S} \pi_{2}{ }^{*}$. No notable shift of the TRPES signal is observed between columns 4 and 5, suggesting the assignment to one of the two ${ }^{3} \pi \pi^{*}$ states $\left({ }^{3} \pi_{\mathrm{S}} \pi_{2}{ }^{*}\right.$ or $\left.{ }^{3} \pi_{5} \pi_{6}{ }^{*}\right)$, which were predicted to occur at similar electron binding energies as the ${ }^{1} n_{S} \pi_{2}{ }^{*}$ state. ${ }^{56}$ We therefore propose internal conversion from the ${ }^{1} \pi_{\mathrm{S}} \pi_{6}{ }^{*}$ to the ${ }^{1} n_{S} \pi_{2}{ }^{*}$ followed by ISC to the ${ }^{3} \pi \pi^{*}$ states as the dominant path for relaxation.

In a simplified picture, we assume a $\Delta \nu=0$ propensity rule, such that any vibrational excitation in the electronically excited states is carried into the cationic state upon photoionization. As shorter excitation wavelengths are employed, photoexcitation to the ${ }^{1} \pi_{\mathrm{S}} \pi_{6}{ }^{*}\left(\mathrm{~S}_{2}\right)$ or ${ }^{1} \pi_{\mathrm{S}} \pi_{2}{ }^{*}\left(\mathrm{~S}_{4}\right)$ states leads to population of these states with increasing vibrational excitation, which according to our assumption leaves the photoelectron kinetic energy $\left(E_{\text {kin }}\right)$ unaffected. Correspondingly, we observe a shift of the photoelectron bands towards higher electron binding 

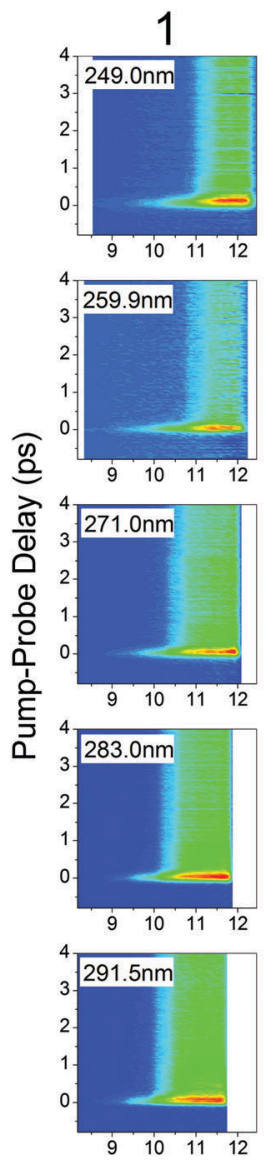

2
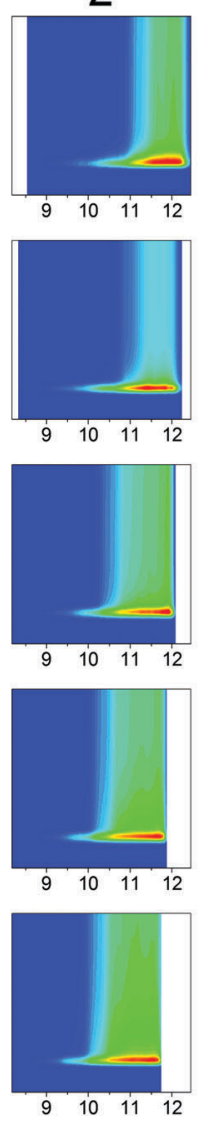

3
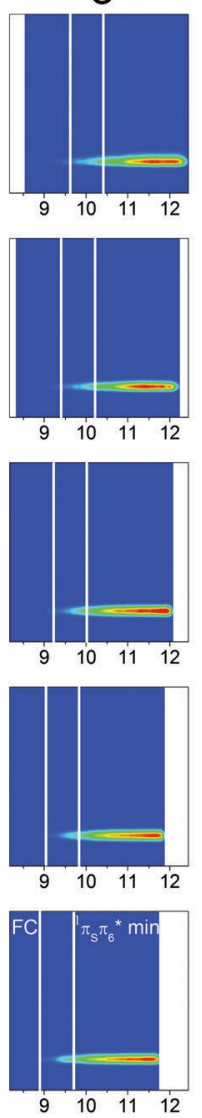

4
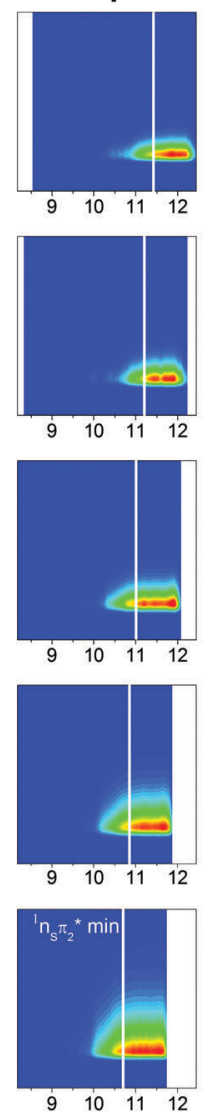

Electron Binding Energy (eV)

5
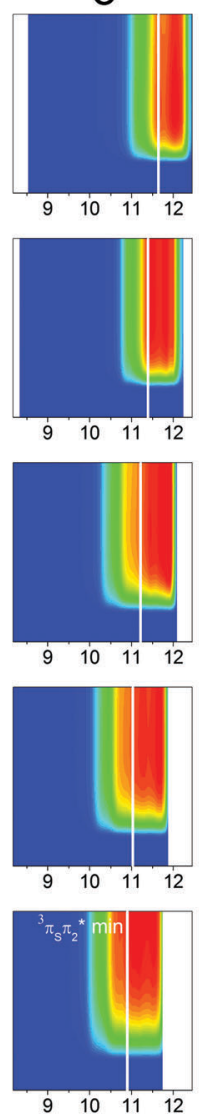
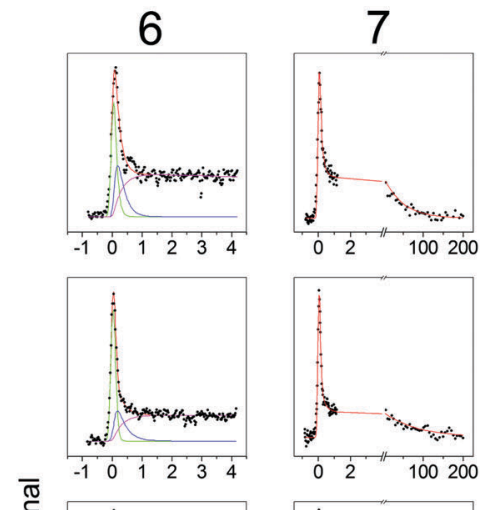

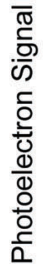
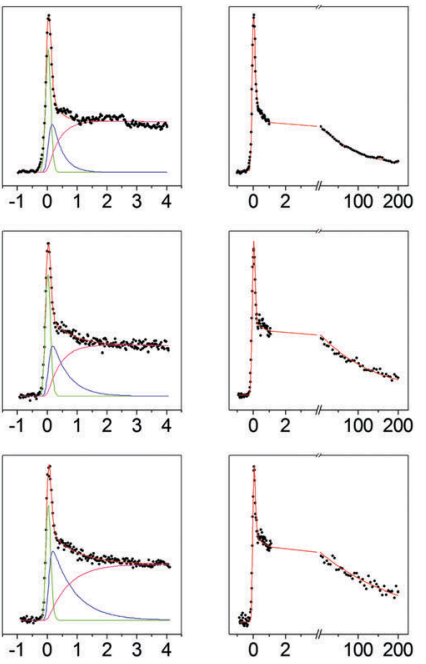

Pump-Probe Delay (ps)

Fig. 3 TRPES and time traces of 2-thiouracil pumped at $249 \mathrm{~nm}$ (first row), $259.9 \mathrm{~nm}$ (second row), $271 \mathrm{~nm}$ (third row), $283 \mathrm{~nm}$ (fourth row) and $291.5 \mathrm{~nm}$ (fifth row) and probed at $329 \mathrm{~nm}$ in a $1+2^{\prime}$ process. TRPES signal, fit, and individual contributions are presented in the first five columns. The vertical white lines indicate calculated total binding energies for different geometries (FC, ${ }^{1} \pi_{\mathrm{S}} \pi_{6}{ }^{*} \min ,{ }^{1} n_{\mathrm{s}} \pi_{2}{ }^{*}$ min, ${ }^{3} \pi_{\mathrm{S}} \pi_{2}{ }^{*}$ min) from ref. 33 , shifted accordingly in the first four rows. Column 6 shows the energy-integrated signals of the first five columns, i.e. the signal (dots), fit (red solid line) and individual contributions (green, blue, pink solid lines) for the short scan. Column 7 shows the signal (dots) and fit (red solid line) for a separate long-range scan, which was used to extract the long-lived decay time constant. The $291.5 \mathrm{~nm}$ data set in the bottom row was previously published in ref. 33 .

energy $\left(E_{\text {bind }}=E_{\text {exc }}+2 E_{\text {probe }}-E_{\text {kin }}\right)$ with increasing excitation energy. The expected shifts are calculated relative to the $291.5 \mathrm{~nm}$ data set described above and indicated by solid white lines throughout Fig. 3. The effect becomes most visible when comparing the TRPES measurements with $291.5 \mathrm{~nm}(\sim 4.25 \mathrm{eV})$ and $249 \mathrm{~nm}(\sim 4.98 \mathrm{eV})$ excitation, where the difference in vibrational excitation amounts to $\sim 0.7 \mathrm{eV}$. Looking at the TRPES contributions in columns 3,4 and 5 , the onset of each photoelectron band is observed to shift between $0.5-1 \mathrm{eV}$ with this change in excitation wavelengths. Due to the good agreement with the expected value and the absence of any other significant wavelength-dependent changes in the TRPES spectra, we conclude that relaxation primarily proceeds along the proposed ${ }^{1} \pi_{\mathrm{S}} \pi_{6}{ }^{*} \rightarrow{ }^{1} \pi_{\mathrm{S}} \pi_{2}{ }^{*} \rightarrow{ }^{3} \pi \pi^{*}$ pathway for all excitation wavelengths employed in this study. For shorter excitation wavelengths, as mentioned above, it is also conceivable that the second bright state $\left({ }^{1} \pi_{\mathrm{S}} \pi_{2}{ }^{*}\right)$ is initially excited, but it can be assumed that this state internally converts to the lower ${ }^{1} \pi_{\mathrm{S}} \pi_{6}{ }^{*}$ state and subsequently to the ${ }^{1} n_{S} \pi_{2}{ }^{*}$ within the temporal resolution of the experiments, as given by the fitted constant $\tau_{1}$.
Table 1 collates all extracted time constants for the threestep relaxation process sorted by increasing excitation wavelength. The time constants $\tau_{1}, \tau_{2}$, and $\tau_{3}$ are associated with the

Table 1 Decay constants of the individual channels with $\tau_{1}$ associated with the relaxation from the initially populated ${ }^{1} \pi \pi^{*}$ states to the ${ }^{1} n \pi^{*}$ state, $\tau_{2}$ to the population of the triplet state via ISC from the ${ }^{1} n \pi^{*}$ state, and $\tau_{3}$ to the relaxation to the ground state. $\tau_{1}$ and $\tau_{2}$ are extracted from a $2 \mathrm{D}$ fit to the short range scan with $\tau_{3}$ fixed and $\tau_{3}$ is extracted from a global fit to the energy-integrated short and long range TRPES scans. An error of $220 \%$ is estimated based on fitting statistics that take into account the reproducibility of data sets and our error in timing calibrations

\begin{tabular}{llclc}
\hline Pump wavelength (nm) & Pump energy $(\mathrm{eV})$ & $\tau_{1}{ }^{a}(\mathrm{fs})$ & $\tau_{2}{ }^{b}(\mathrm{fs})$ & $\tau_{3}^{c}(\mathrm{ps})$ \\
\hline 249.0 & 4.98 & 85 & 207 & 48.1 \\
259.9 & 4.77 & 67 & 285 & 85.6 \\
271.0 & 4.58 & $<50$ & 333 & 109 \\
283.0 & 4.38 & $<50$ & 544 & 142 \\
291.5 & 4.25 & $<50$ & 775 & 203
\end{tabular}

${ }^{a}$ Assigned to internal conversion from the ${ }^{1} \pi_{\mathrm{S}} \pi_{6}{ }^{*}$ to ${ }^{1} \mathrm{n}_{\mathrm{S}} \pi_{2}{ }^{*}$ state. ${ }^{b}$ Assigned to intersystem crossing from the ${ }^{1} \mathrm{n}_{\mathrm{S}} \pi_{2}{ }^{*}$ state to the triplet manifold. ${ }^{c}$ Assigned to the decay of the lowest-energy triplet state. 
TRPES contributions plotted in columns 3, 4, and 5, respectively. A general trend can be observed, where excitation with higher photon energy results in faster relaxation dynamics for $\tau_{2}$ and $\tau_{3}$. For all wavelengths, the first time constant $\tau_{1}$ is ultrafast $(<100 \mathrm{fs})$ and within our Gaussian instrument response function. The second time constant $\tau_{2}$ ranges from ca. 200 to 800 fs and the third constant $\tau_{3}$ from ca. 50 to $200 \mathrm{ps.}$

Comparing our time constants to the dynamics studies of Mai et al., ${ }^{37}$ a good agreement between theory and experiments is observed. According to their mechanism, the presence of a ${ }^{1} \pi_{\mathrm{S}} \pi_{6}{ }^{*} /{ }^{1} \mathrm{n}_{\mathrm{S}} \pi_{2}{ }^{*}$ conical intersection close to the ${ }^{1} \pi_{\mathrm{S}} \pi_{6}{ }^{*}$ minimum promotes ultrafast $(<100 \mathrm{fs})$ internal conversion following excitation to the bright ${ }^{1} \pi_{\mathrm{S}} \pi_{6}{ }^{*}$ state. The slight increase in $\tau_{1}$ at shorter excitation wavelengths may be attributable to population of the higher-lying ${ }^{1} \pi_{\mathrm{S}} \pi_{2}{ }^{*}$ state and consequently a longer time to populate the ${ }^{1} \mathrm{n}_{\mathrm{S}} \pi_{2}{ }^{*}$ state via the ${ }^{1} \pi_{\mathrm{S}} \pi_{6}{ }^{*}$. Importantly, the state switch to ${ }^{1} \mathrm{n}_{\mathrm{S}} \pi_{2}{ }^{*}$ induces changes in the geometry of 2-thiouracil, like out-of-plane puckering of the $\mathrm{C}_{2}$ atom and pyramidalization of the thiocarbonyl group, which take hundreds of femtoseconds to reach the ${ }^{1} n_{S} \pi_{2}{ }^{*}$ minimum. At that point, population transfer to the ${ }^{3} \pi_{\mathrm{S}} \pi_{2}{ }^{*}$ state can occur after surmounting a small energy barrier $\left(0.05-0.09 \mathrm{eV}^{36}\right)$ to reach the ${ }^{1} n_{\mathrm{S}} \pi_{2}{ }^{*} /{ }^{3} \pi_{\mathrm{S}} \pi_{2}{ }^{*}$ intersection seam. This barrier is possibly the reason for the observed pump-wavelength dependence of the ISC time constant. At shorter pump wavelengths, it is also conceivable that a minor fraction of the population takes other ISC routes, e.g., ${ }^{1} \pi_{\mathrm{S}} \pi_{2}{ }^{*} \rightarrow{ }^{3} \mathrm{n}_{\mathrm{S}} \pi_{2}{ }^{*} \rightarrow{ }^{3} \pi_{\mathrm{S}} \pi_{2}{ }^{*}$, as observed in the dynamics simulations of Mai et al. ${ }^{37}$ These additional ISC routes would also contribute to the observed increase of the ISC rate.

The third time constant $\tau_{3}$ is associated with ISC from the lowest adiabatic triplet potential energy surface, which features two distinct minima arising from the ${ }^{3} \pi_{\mathrm{S}} \pi_{2}{ }^{*}$ and ${ }^{3} \pi_{5} \pi_{6}{ }^{*}$ triplet states, back to the ground state. The associated decay time of tens to hundreds of picoseconds may be unexpectedly fast, but is theoretically supported by the presence of only a small energy barrier $(0.2 \mathrm{eV})$ at the ${ }^{3} \pi_{\mathrm{S}} \pi_{2}{ }^{*} / \mathrm{S}_{0}$ crossing point and the magnitude of the SOCs $\left(130 \mathrm{~cm}^{-1}\right)$ between the triplet state and the ground state. ${ }^{33}$ In contrast, as discussed in a previous work, ${ }^{33}$ the natural bases uracil and thymine show triplet lifetimes of $>1 \mathrm{~ns}$ due to much smaller SOCs $\left(\sim 2 \mathrm{~cm}^{-1}\right)$ and larger energy barriers $(\sim 0.4 \mathrm{eV}) .{ }^{33}$ Like for $\tau_{2}$, the presence of an energy barrier to reach the triplet-singlet intersection seam could be the reason for the observed strong excitation wavelength-dependence of $\tau_{3}$.

Collectively, geometric changes of the molecule and the energy barriers to access the crossing seams play an important role in its ability to reach crossing points between excited states. Photoexcitation at shorter excitation wavelengths results in highly vibrationally excited molecules, leading to large-amplitude out-of-plane vibrations, including the carbon puckering and sulfur pyramidalization modes necessary for the ISC processes, and allows surmounting the relevant barriers, as described above. This reasoning can explain the systematic trend in time constants observed in the TRPES data.

\section{Role of the excitation wavelength in the ultrafast excited-state} dynamics in solution

The gas-phase results indicate that the magnitude of the ISC lifetimes to populate the triplet state or to repopulate the ground state decrease by a factor of four as the excitation wavelength is decreased from 291.5 to $249 \mathrm{~nm}(4.25$ to $4.98 \mathrm{eV})$. It is proposed that this decrease is due to a higher probability of the population to overcome the calculated energy barriers to access the ${ }^{1} n_{S} \pi_{2}{ }^{*} / \pi_{S} \pi_{2}{ }^{*}$ and the ${ }^{3} \pi_{S} \pi_{2}{ }^{*} / S_{0}$ intersection seams. Recent experimental and computational studies ${ }^{19,21,31,33-37}$ suggest that the electronic relaxation mechanism of 2-thiouracil in solution is similar to that in the gas phase, which motivated further TRAS studies for direct comparison. In this section, we present the effects of varying the excitation wavelength on the ultrafast (i.e., sub-ns) dynamics of 2-thiouracil in solution, whereas the effects of excitation wavelength on the triplet decay to the ground state are postponed to the next section.

Fig. 4 shows the transient absorption contour plots, the decay associated spectra (DAS), kinetic traces at selected probe wavelengths, and the corresponding fitting curves for 2-thiouracil in acetonitrile upon excitation at 268, 290, and $316 \mathrm{~nm}$. Lifetimes obtained from a global fit analysis of the broadband data using a two-component sequential model plus a constant offset (see ESI $\dagger$ for details) are presented in Table 2 . The black DAS is present at time zero and has been previously assigned to the ${ }^{1} n_{S} \pi_{2}{ }^{*}$ state, ${ }^{31}$ but may also capture some residual absorption of the very shortlived ${ }^{1} \pi_{\mathrm{S}} \pi^{*}$ states. The black DAS subsequently evolves to the red spectrum with $\tau_{1}^{\text {solv }}$, which further evolves to a constant offset spectrum (in blue) with $\tau_{2}^{\text {solv }}$. The latter spectrum does not decay within the 200 ps time window used in the fs-TRAS experiments and was previously identified as the absorption spectrum of the long-lived ${ }^{3} \pi \pi^{*}$ state of 2 -thiouracil in acetonitrile. ${ }^{19,31}$ Its ns decay dynamics will be discussed separately in the next section.

Inspection of Fig. 4 and Table 2 shows subtle, but noticeable spectral and temporal changes in the excited-state dynamics of 2-thiouracil in solution as the excitation wavelength is varied (all spectral changes mentioned in the following correspond to a decrease in excitation wavelength). The spectral changes may be more conveniently visualized in the normalized DAS shown in Fig. S3 in the ESI. $\dagger$ The DAS show that the spectrum of the ${ }^{1} \mathrm{n}_{\mathrm{S}} \pi_{2}{ }^{*}$ state (black spectrum) broadens and slightly redshifts. Similarly, the intermediate spectrum depicted in red broadens and its absorption maximum redshifts from $c a$. 682 to $\geq 700 \mathrm{~nm}$ (see transient absorption spectra in Fig. S2, ESI $\dagger$ ). The maximum of the ${ }^{3} \pi \pi^{*}$ state spectrum depicted in blue also redshifts slightly. Upon excitation at $316 \mathrm{~nm}$, the intermediate spectrum in red decays across the entire spectral probe window to the spectrum assigned to the ${ }^{3} \pi \pi^{*}$ state (blue spectrum). Conversely, as the excitation wavelength is decreased from $316 \mathrm{~nm}$ to 290 or $268 \mathrm{~nm}$, the redshift of the intermediate absorption spectrum in red leads to an apparent increase in absorbance in the spectral range from 625 to $700 \mathrm{~nm}$ before reaching the spectrum of the ${ }^{3} \pi \pi^{*}$ state. The redshifting and broadening of these two DAS suggest that the excited-state absorption may be occurring from high-energy parts of the corresponding potential energy surfaces. In terms of the lifetimes, a slight increase in the magnitude of 
1
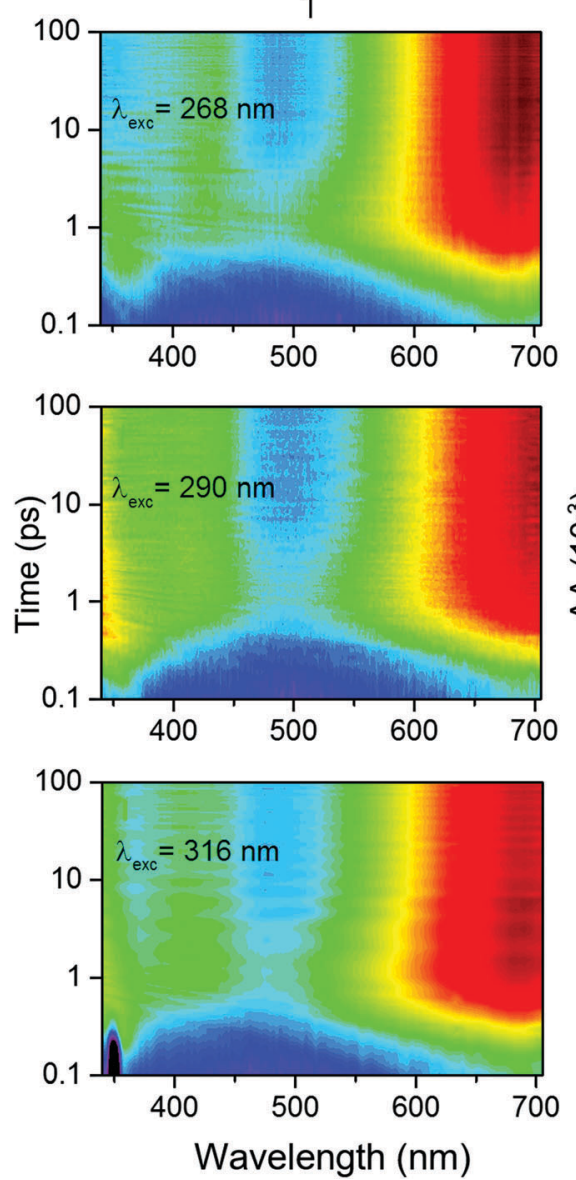

2

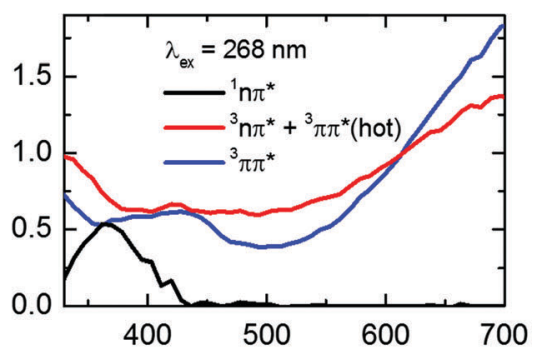

3

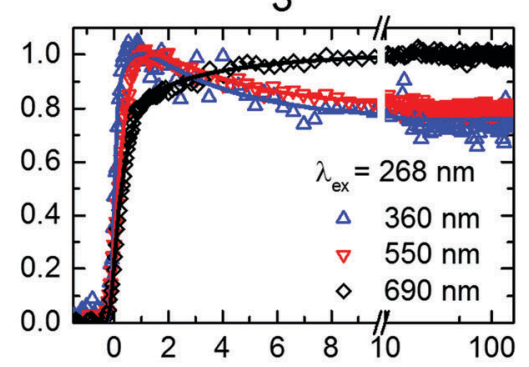

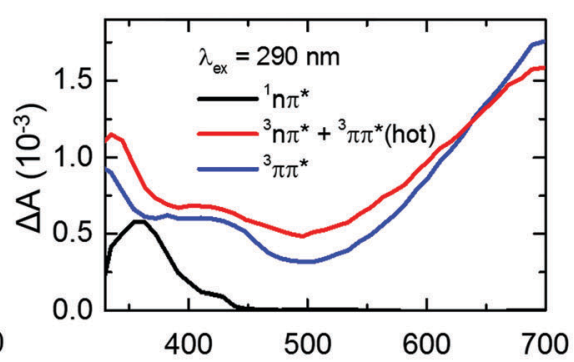
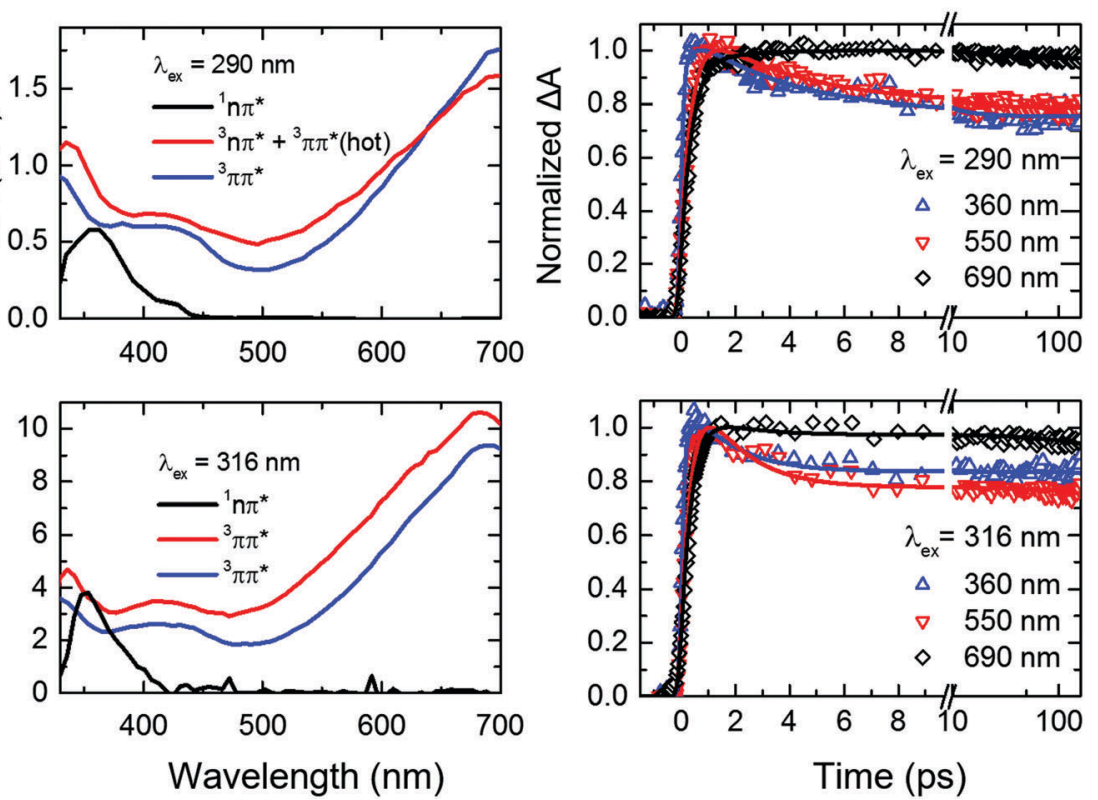

Fig. 4 (Column 1) Contour plots of the multidimensional sub-ns TRAS data collected for 2 -thiouracil in acetonitrile following excitation at the specified wavelengths. For clarity and completeness, the experimental transient absorption data is shown in Fig. S2 in the ESI. $\dagger$ The data was globally fitted to a two-component sequential kinetic model plus a constant offset, producing the decay associated spectra (DAS, Column 2) and the kinetic traces shown at representative probe wavelengths (Column 3). The black DAS spectrum is present at time zero and evolves to the red spectrum with $\tau_{1}^{\text {solv }}$, which further evolves to an offset (spectrum in blue) with $\tau_{2}^{\text {solv }}$. These lifetimes are gathered in Table 2 . In the bottom middle panel, the change in amplitude between the blue and red DAS (both assigned to the ${ }^{3} \pi \pi^{*}$ state) is due triplet self-quenching (see main text for details).

Table 2 Excited-state lifetimes of 2-thiouracil in acetonitrile as a function of excitation wavelength based on the fs-TRAS and ns-TRAS (next section) experiments

\begin{tabular}{lllc}
\hline$\lambda_{\mathrm{ex}}(\mathrm{nm})$ & $\tau_{1}^{\text {solv } a}(\mathrm{fs})$ & $\tau_{2}^{\text {solv }}(\mathrm{ps})$ & $\tau_{3}^{\text {solv } d}(\mathrm{~ns})$ \\
\hline 268 & $440 \pm 30$ & $3.7 \pm 0.2^{b}$ & $73 \pm 10$ \\
290 & $380 \pm 30$ & $3.5 \pm 0.2^{b}$ & $69 \pm 4$ \\
316 & $330 \pm 40$ & $5.5 \pm 0.7^{c}$ & $296 \pm 23$
\end{tabular}

${ }^{a}$ Assigned to a combination of internal conversion from the ${ }^{1} \pi_{\mathrm{S}} \pi_{6}{ }^{*}$ to ${ }^{1} n_{\mathrm{S}} \pi_{2}{ }^{*}$ state and intersystem crossing to the triplet manifold. ${ }^{b}$ Assigned to ${ }^{3} \mathrm{n} \pi^{*}$ and vibrational cooling in the $\mathrm{T}_{1}$ state following 268 and $290 \mathrm{~nm}$ excitation. ${ }^{c}$ Assigned to triplet self-quenching following $316 \mathrm{~nm}$ excitation. ${ }^{d}$ Triplet decay lifetimes measured in a separate experiment (see below) for a 2-thiouracil concentration of $0.6 \mathrm{mM}$; reported uncertainties are twice the standard error obtained from global analyses of at least two independent data sets of experiments at each excitation wavelength.

$\tau_{1}^{\text {solv }}$ is observed with a decrease in excitation wavelength (see also Fig. 5), whereas the magnitude of $\tau_{2}^{\text {solv }}$ also changes (Table 2).

Based on the discussion above and the assignment of the first and second DAS (black and red in Fig. 4), we assign $\tau_{1}^{\text {solv }}$ to the ${ }^{1} \pi_{\mathrm{S}} \pi_{6}{ }^{*} \rightarrow{ }^{1} \mathrm{n}_{\mathrm{S}} \pi_{2}{ }^{*}$ (hot) $\rightarrow{ }^{1} \mathrm{n}_{\mathrm{S}} \pi_{2}{ }^{*} \rightarrow{ }^{3} \pi_{\mathrm{S}} \pi_{2}{ }^{*}$ process, ${ }^{19,31}$ thus this constant is approximately equivalent to a combination of $\tau_{1}$ and $\tau_{2}$ of the TRPES experiments. The slight increase in the magnitude of $\tau_{1}^{\text {solv }}$ and the broadening in the first DAS may be due to increased vibrational energy in the ${ }^{1} n_{S} \pi_{2}{ }^{*}$ state, as vibrational cooling dynamics in an electronic state exhibits longer cooling times and broader electronic absorption spectra as the excitation energy is increased. ${ }^{57}$ Hence, the slowdown in $\tau_{1}^{\text {solv }}$ with decreasing excitation wavelength can be understood as due to the vibrational energy redistribution (intramolecular, intermolecular, or both) being the rate-limiting step. However, another possibility is that the change in $\tau_{1}^{\text {solv }}$ may be due to excitation of the higher-energy ${ }^{1} \pi_{\mathrm{s}} \pi_{2}{ }^{*}$ state in addition to (or at the expense of) the ${ }^{1} \pi_{\mathrm{s}} \pi_{6}{ }^{*}$ state. As shown in ref. 36 and 37 , population in ${ }^{1} \pi_{\mathrm{s}} \pi_{2}{ }^{*}$ needs to overcome a small energy barrier before internal conversion to the ${ }^{1} n_{S} \pi_{2}{ }^{*}$ state can occur (in contrast to population in the ${ }^{1} n_{S} \pi_{6}{ }^{*}$ state), which could also explain the increase in the magnitude of $\tau_{1}^{\text {solv }}$. If so, the observed broadening in the ${ }^{1} n_{\mathrm{S}} \pi_{2}{ }^{*}$ state spectrum could alternatively be explained by the absorption of the ${ }^{1} \pi_{\mathrm{S}} \pi_{2}{ }^{*}$ and/or 


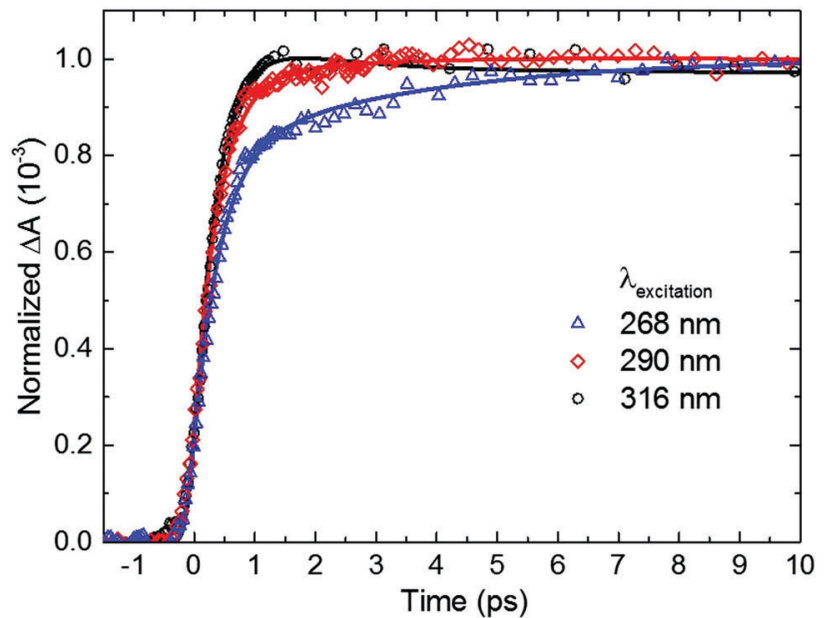

Fig. 5 Dependence of the rate of triplet state population on excitation wavelength for 2 -thiouracil in acetonitrile. Kinetic traces were taken at a probe wavelength of $690 \mathrm{~nm}$, near the absorption maximum of the triplet state. For easier inspection, only the initial 10 ps time window of the dynamics is shown (see Fig. 4, right panel for an extended time window).

${ }^{1} \pi_{\mathrm{S}} \pi_{6}{ }^{*}$ states in the same spectral probe region that the ${ }^{1} n_{S} \pi_{2}{ }^{*}$ state absorbs. Calculations of the absorption spectra of these states reported in the ESI $\dagger$ (Fig. S4) lend support to this proposition.

The spectral and temporal changes associated with $\tau_{2}^{\text {solv }}$ as the excitation wavelength is decreased are more challenging to interpret. Upon $316 \mathrm{~nm}$ excitation, a uniform decay of absorbance from the red to the blue DAS is seen across the entire spectral window (Fig. 4). This decay process has been previously $^{31}$ assigned to ground-state repopulation due to triplet self-quenching of 2-thiouracil molecules that aggregated in the ground state prior to excitation..$^{19,31,58}$ In other words, a fraction of the triplet state population decays to the ground state by triplet self-quenching during $\tau_{2}^{\text {solv }}$ when excitation is done at $316 \mathrm{~nm}$. On the other hand, for excitation at 268 and $290 \mathrm{~nm}$, an increase in absorbance at probe wavelengths of $c a$. $\geq 625 \mathrm{~nm}$ is observed in going from the second DAS (red spectrum) to the third DAS (blue), such that the latter DAS reaches the triplet absorption maximum at around $700 \mathrm{~nm}$. Taking into consideration all the experimental and computational data available to date, ${ }^{31,33-37}$ we assign $\tau_{2}^{\text {solv }}$ upon 268 or $290 \mathrm{~nm}$ excitation to vibrational cooling dynamics in the triplet state-analogous to 2-thiothymine. ${ }^{59}$ The narrowing of the transient absorption spectra during this lifetime supports this assignment. The different observations when excitation is performed at 290 or $268 \mathrm{~nm}$ versus at $316 \mathrm{~nm}$ can be explained by the different amount of vibrational energy in the triplet state. Additionally, we remark that a fraction of the excess vibrational energy may also redistribute intra-molecularly, in which case it could be used to overcome the energy barrier between the triplet minimum and the ground state intersection seam. This latter statement is explored in more detail in the next section from both experimental and computational perspectives.

\section{Role of the excitation wavelength in the triplet state decay} lifetime in solution

As stated in the previous section, any fraction of the excess excitation energy that accumulates intra-molecularly in the triplet state may in principle be used to overcome the calculated energy barrier between the triplet minimum and the ground state intersection seam. ${ }^{33,36,37}$ If so, an increase in the rate of triplet state decay back to the ground state may be observed, similar to that reported above in the gas phase. Hence, nanosecond TRAS experiments monitoring the decay of the triplet state of 2-thiouracil were performed in acetonitrile at multiple excitation wavelengths, in order to provide more insights into this possibility.

From the fitting of the decay traces (see Fig. 6), it is apparent that triplet state decay in solution is three orders of magnitude slower than in gas phase. Moreover, the decay of the triplet state $\left(\tau_{3}^{\text {solv }}\right.$ in Table 2 ) is fivefold faster following excitation at 268 or $290 \mathrm{~nm}$ than at $316 \mathrm{~nm}$. These experimental observations support the idea that an energy barrier between the triplet minimum and the ground state intersection seam also exists in solution.

PCM-MS-CASPT2(12,9)/cc-pVDZ calculations (Fig. 7) evidence that an energy barrier does indeed exist between the triplet minimum and the intersection seam with the ground state in acetonitrile. As shown in Fig. 7, the magnitude of the energy barrier increases by $\sim 40 \%$, from $0.20 \mathrm{eV}$ in vacuum to about $0.28 \mathrm{eV}$ in acetonitrile, which partially explains the much slower triplet state decay in solution. Furthermore, the calculations predict that the lowest triplet surface has two minima, with the second triplet minimum $\left({ }^{3} \pi_{5} \pi_{6}{ }^{*}\right)$ being slightly lower in energy than the ${ }^{3} \pi_{\mathrm{S}} \pi_{2}{ }^{*}$ minimum by $c a$. $0.1 \mathrm{eV}$ in acetonitrile. The ${ }^{3} \pi_{5} \pi_{6}{ }^{*}$ minimum is connected to a second $T_{1} / S_{0}$ intersection seam $\left({ }^{3} \pi_{5} \pi_{6} * / S_{0}\right)$, where carbon atom $C_{5}$ is strongly displaced from the molecular plane. This intersection seam is analogous to the one found in the natural nucleobases thymine and uracil, ${ }^{33}$ but its high energy and negligible SOCs mean that decay at this seam will not be competitive with decay at the

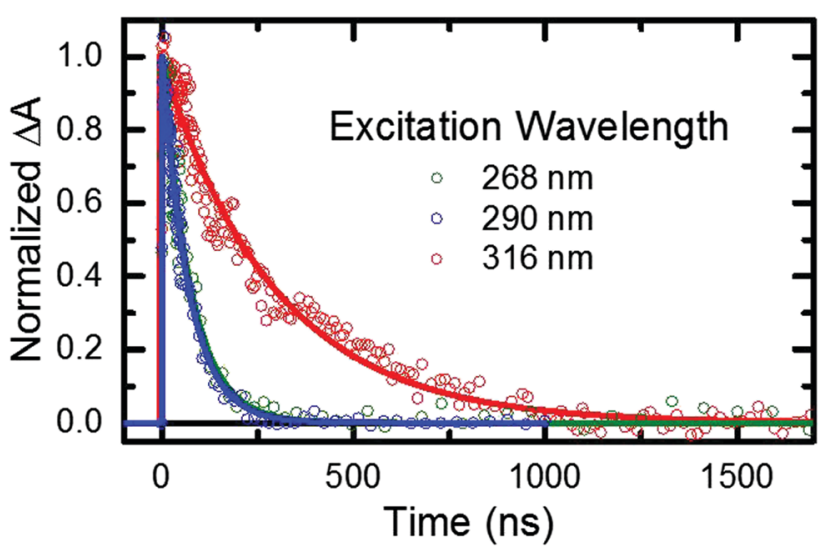

Fig. 6 Triplet state decay traces of 2-thiouracil at $0.6 \mathrm{mM}$ in $\mathrm{N}_{2}$-saturated acetonitrile following excitation at 268, 290, or $316 \mathrm{~nm}$. Each trace is an average across the triplet absorption band in the probe spectral range from ca. 630 to $710 \mathrm{~nm}$ 
${ }^{3} \pi_{\mathrm{S}} \pi_{2}{ }^{*} / \mathrm{S}_{0}$ seam. Hence, ISC to the ground state should always involve the ${ }^{3} \pi_{\mathrm{S}} \pi_{2}{ }^{*} / \mathrm{S}_{0}$ intersection, independent of the minimum to which the population has settled during the vibrational cooling process. Population trapped in the lower-energy ${ }^{3} \pi_{5} \pi_{6}{ }^{*}$ minimum will therefore have to overcome an effective barrier of $0.36 \mathrm{eV}$ to undergo ISC to the ground state. We think that this theoretical prediction can satisfactorily explain the observation that excitation at $316 \mathrm{~nm}$ leads to an ISC lifetime of $c a .300 \mathrm{~ns}$, whereas excitation at shorter wavelengths than $c a .308 \mathrm{~nm}^{32}$ results in an ISC lifetime of $c a .70$ ns (see Fig. 6 and Table 2). Excitation at $316 \mathrm{~nm}$ leads to trapping of population $\left(\tau_{3}^{\text {solv }} \sim 300 \mathrm{~ns}\right)$ in the ${ }^{3} \pi_{5} \pi_{6}{ }^{*}$ minimum, whereas excitation at wavelengths shorter than $308 \mathrm{~nm}$ may provide enough excess vibrational energy to settle in the ${ }^{3} \pi_{\mathrm{S}} \pi_{2}{ }^{*}$ minimum. Since the ${ }^{3} \pi_{\mathrm{S}} \pi_{2}{ }^{*}$ minimum is predicted to access the $T_{1} / S_{0}$ crossing more effectively than the ${ }^{3} \pi_{5} \pi_{6}{ }^{*}$ minimum (Fig. 7), excitation at shorter wavelengths can populate the ${ }^{3} \pi_{\mathrm{S}} \pi_{2}{ }^{*}$ minimum more effectively, leading to a faster decay of the triplet state population. If this interpretation of the experimental results holds true, then the DAS assigned to the lowest-energy triplet state in Fig. 4 could represent a weight-averaged linear combination of the spectra of the ${ }^{3} \pi_{\mathrm{S}} \pi_{2}{ }^{*}$ and the ${ }^{3} \pi_{5} \pi_{6}{ }^{*}$ minima, particularly as the excitation energy is increased. This could explain the subtle, but noticeable spectral changes in the DAS assigned to the lowest-energy triplet

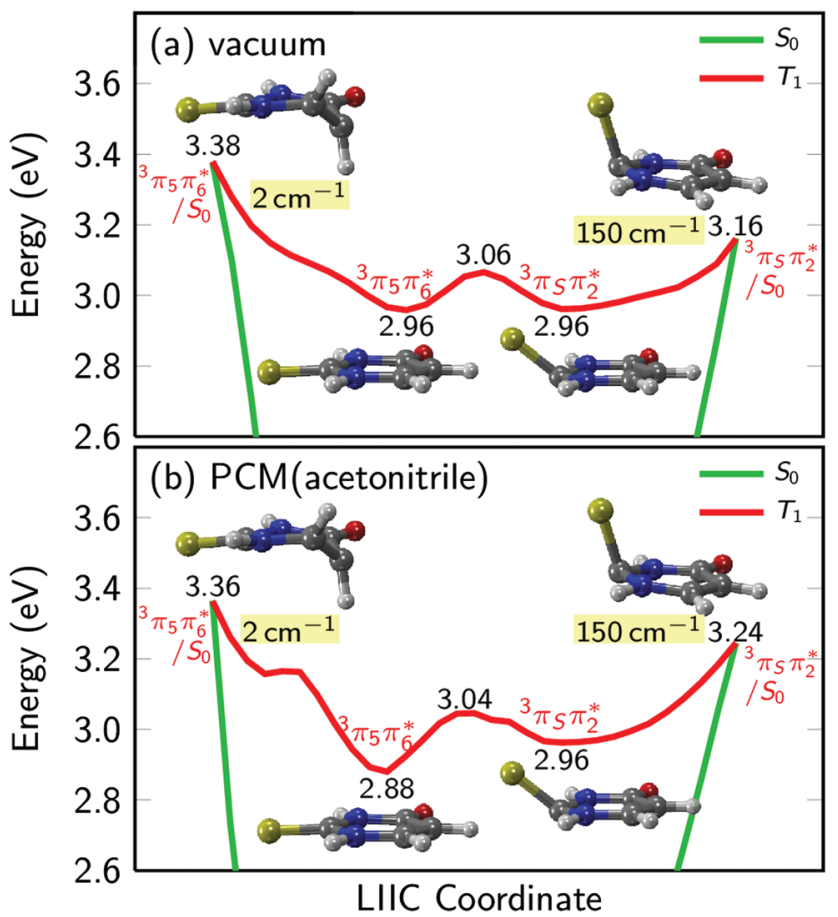

Fig. 7 Potential energy profiles of the lowest triplet state of 2-thiouracil in vacuum and acetonitrile. Numbers in labels give the energy in eV above the ground state minimum, numbers shaded in yellow give the magnitude of SOCs at the crossing points. In vacuum and solution, the lowest triplet potential energy surface has two minima, arising from the ${ }^{3} \pi_{\mathrm{s}} \pi_{2}{ }^{*}$ and ${ }^{3} \pi_{5} \pi_{6}{ }^{*}$ states, respectively. The minima are separated by a small barrier and are each connected to a $T_{1} / S_{0}$ crossing point. The results are computed with MS-CASPT2(12,9)/cc-pVDZ as in ref. 37. For panel (b), the PCM charges were computed in equilibrium with the $T_{1}$ state. state with excitation energy (see the normalized DAS in Fig. S3 in the $\mathrm{ESI} \dagger)$.

\section{Excitation-wavelength-dependent dynamics: gas-phase versus condensed-phase}

As explained above, the excited-state dynamics of 2-thiouracil exhibits a striking dependence on excitation wavelength. In both gas and condensed phases, a decrease in excitation wavelength allows for population of higher-lying singlet states and for the accumulation of excess vibrational energy in the lowest-energy singlet state $\left({ }^{1} n_{S} \pi_{2}{ }^{*}\right)$. Interestingly, the excess vibrational energy in the ${ }^{1} \mathrm{n}_{\mathrm{S}} \pi_{2}{ }^{*}$ state apparently modulates the rate of intersystem crossing in the gas and solution phases differently. In the gas phase, more excitation energy accelerates ISC to the triplet manifold as population in the ${ }^{1} n_{S} \pi_{2}{ }^{*}$ can more easily overcome the energy barrier to the ${ }^{1} n_{S} \pi_{2}{ }^{*} / \pi_{S} \pi_{2}{ }^{*}$ intersection seam. In solution, it appears that the rate-limiting step of the experimentally-measured ISC lifetime $\left(\tau_{1}^{\text {solv }}\right.$, which combines the following processes: ${ }^{1} \pi_{\mathrm{S}} \pi_{6}{ }^{*} \rightarrow{ }^{1} \mathrm{n}_{\mathrm{S}} \pi_{2}{ }^{*}$ (hot) $\rightarrow$ ${ }^{1} n_{\mathrm{S}} \pi_{2}{ }^{*} \rightarrow{ }^{3} \pi_{\mathrm{S}} \pi_{2}{ }^{*}$ ) is the dissipation of excess vibrational energy in the ${ }^{1} n_{\mathrm{S}} \pi_{2}{ }^{*}$ state (either by intramolecular or intermolecular redistribution, or both), instead of overcoming the energy barrier to the ${ }^{1} n_{S} \pi_{2}{ }^{*} / \pi_{S} \pi_{2}{ }^{*}$ intersection seam, as is the case in the gas phase. This supposition can satisfactorily explain the observation that while ISC in the gas phase occurs more rapidly with decreasing excitation wavelength, in the condensed phase ISC $\left(\tau_{1}^{\text {solv }}\right)$ slightly slows down. In other words, while the ISC lifetime in the gas phase is primarily modulated by the amount of excess vibrational energy available to overcome the relevant energy barrier, in solution, the ISC lifetime is primarily modulated by the rate of vibrational energy redistribution in the ${ }^{1} n_{S} \pi_{2}{ }^{*}$ (hot) state. As remarked above, vibrational dynamics in an electronic state usually exhibit longer lifetimes as the excitation wavelength is decreased. ${ }^{57}$

The triplet state decay to the ground state occurs more rapidly with decreasing excitation wavelength in both the gas and condensed phases. However, this dependence is bimodal $\left(\tau_{3}^{\text {solv }}=300 \mathrm{~ns}\right.$ with $\lambda_{\mathrm{ex}}=316 \mathrm{~nm}$ and $\tau_{3}^{\text {solv }}=70 \mathrm{~ns}$ when $\lambda_{\mathrm{ex}} \leq$ $308 \mathrm{~nm}$ ) in solution, whereas in gas phase $\tau_{3}$ depends continuously on the excitation wavelength. On the one hand, Fig. 7 shows that $0.2 \mathrm{eV}$ are needed to reach the ${ }^{3} \pi_{\mathrm{S}} \pi_{2}{ }^{*} / \mathrm{S}_{0}$ crossing from either of the calculated $\mathrm{T}_{1}$ minima in the gas phase, and this barrier can be more easily overcome as more excess vibrational energy is available. This process leads to the trend observed for the triplet decay reported in Table 1. On the other hand, the transfer of excess vibrational energy to the solvent in the triplet manifold seems to occur faster than triplet decay in acetonitrile, which leads to trapping of the system in either of the calculated $\mathrm{T}_{1}$ minima, with the distribution depending on the amount of excess energy initially available. The removal of excess vibrational energy by the solvent most likely explains the three orders of magnitude slower triplet decay in solution compared to vacuum. Furthermore, in acetonitrile, the ${ }^{3} \pi_{5} \pi_{6}{ }^{*}$ and ${ }^{3} \pi_{\mathrm{S}} \pi_{2}{ }^{*}$ minima are not degenerate (Fig. 7) and therefore, whether population in the $\mathrm{T}_{1}$ state relaxes to the ${ }^{3} \pi_{5} \pi_{6}{ }^{*}$ or to the ${ }^{3} \pi_{\mathrm{S}} \pi_{2}{ }^{*}$ minimum will affect how fast the triplet state can be 
depopulated. Specifically, population in the ${ }^{3} \pi_{5} \pi_{6}{ }^{*}$ minimum must overcome an energy barrier of $0.36 \mathrm{eV}$ to access the ${ }^{3} \pi_{\mathrm{S}} \pi_{2}{ }^{*} / \mathrm{S}_{0}$ crossing, whereas population in the ${ }^{3} \pi_{\mathrm{S}} \pi_{2}{ }^{*}$ minimum only needs to surmount an energy barrier of $0.28 \mathrm{eV}$. Thus, excitation with wavelengths shorter than $308 \mathrm{~nm}$ seems to populate primarily the ${ }^{3} \pi_{\mathrm{S}} \pi_{2}{ }^{*}$ minimum, whereas excitation at $316 \mathrm{~nm}$ seems to populate mostly the ${ }^{3} \pi_{5} \pi_{6}{ }^{*}$ minimum. The latter leads to a slower rate of triplet state decay. It appears that a combination of electronic and vibrational solvent effects leads to a lengthening of the triplet decay lifetime by nearly three orders of magnitude in going from gas phase to acetonitrile.

\section{Conclusions}

TRPES and TRAS experiments reveal that the excited-state dynamics of 2-thiouracil can be modulated sensitively depending on the excitation wavelength used. As the excitation wavelength is decreased, high-lying singlet states can be populated, which leads to the population of the lowest-energy ${ }^{1} n \pi^{*}$ state with increasing excess of vibrational energy in both gas and condensed phases. It is proposed that excitation to higher vibrational levels and, in particular, out-of-plane vibrational modes, promote puckering and pyramidalization distortions in 2-thiouracil that are necessary to access ISC pathways. In the gas phase, the excess vibrational energy is used to overcome an energy barrier to access the triplet state, whereas intermolecular energy transfer in solution removes the excess energy before ISC to the triplet state ensues. Specifically, overcoming the energy barrier to access the ${ }^{1} n_{S} \pi_{2}{ }^{*} /{ }^{3} \pi_{S} \pi_{2}{ }^{*}$ intersection seam controls the ISC lifetime to the triplet state, whereas vibrational energy redistribution of the ${ }^{1} n \pi^{*}$ (hot) state is proposed to be the rate-limiting step in the ISC event to the triplet state in solution.

We have also shown that triplet state decay to the ground state occurs more rapidly as the excitation energy is increased in both gas and condensed phases. Strikingly, this acceleration in the triplet state decay occurs bimodal in solution, whereas it is continuous in the gas phase. This behavior is explained by the presence of two minima in the potential energy surface of the lowest-energy triplet state, together with the existence of an energy barrier to access the ${ }^{3} \pi_{\mathrm{S}} \pi_{2}{ }^{*} / \mathrm{S}_{0}$ crossing seam. While the calculations show that the energy barrier between both triplet minima does not change appreciably in going from vacuum to acetonitrile and both triplet minima have equal energy in the gas phase, the two minima are non-degenerate in solution. This latter observation seems to explain the decay of the triplet state to the ground state in a bimodal fashion in solution, as the excitation wavelength is decreased, while in the gas phase a continuous acceleration is observed. We note that, while our manuscript was under review, another work was published by Bai and Barbatti ${ }^{60}$ where the triplet decay of the closely related 2-thiothymine was investigated theoretically using excited-state optimizations and PES scans in the gas phase. Their results fully agree with our gas phase calculations, also finding the two triplet minima and related crossing points.
In conclusion, the experimental and computational results presented in this study show that the accessibility of conical intersections, crossing seams, and energy barriers along the associated singlet and triplet relaxation coordinates can be better understood via a systematic variation of the excitation wavelength in both gas and condensed phases. Specifically, systematic trends among the intersystem crossing lifetimes were revealed with a decrease in excitation wavelength, which can be directly correlated to the topology of the potential energy surfaces, and satisfactorily interpreted by static $a b$ initio calculations that take into consideration solvents effects and that are complementary to the high-level dynamics simulations carried out in vacuum. ${ }^{37}$

\section{Acknowledgements}

This work was supported by the National Science Foundation, grant CHE-1362237 (J. S.-R., A. M., S. U.), and grants CHE-1255084 and CHE-1539808 (B. A., M. P., C. E. C.-H.), and the Austrian Science Fund FWF, grant P25827 (S. M., P. M., L. G.). J. S.-R., A. M., and S. U. would also like to thank Mr T. J. Godfrey for his assistance in the laboratory. Part of the calculations were performed at the Vienna Scientific Cluster (VSC3). We also thank the COST action XLIC (CM1204) for fruitful discussions over the past years.

\section{References}

1 C. E. Crespo-Hernández, B. Cohen, P. M. Hare and B. Kohler, Chem. Rev., 2004, 104, 1977-2019.

2 A. L. Sobolewski and W. Domcke, Europhys. News, 2006, 37, 20-23.

3 C. T. Middleton, K. de La Harpe, C. Su, Y. K. Law, C. E. Crespo-Hernández and B. Kohler, Annu. Rev. Phys. Chem., 2009, 60, 217-239.

4 B. Kohler, J. Phys. Chem. Lett., 2010, 1, 2047-2053.

5 T. Gustavsson, R. Improta and D. Markovitsi, J. Phys. Chem. Lett., 2010, 1, 2025-2030.

6 K. Kleinermanns, D. Nachtigallová and M. S. de Vries, Int. Rev. Phys. Chem., 2013, 32, 308-342.

7 M. Barbatti, A. C. Borin and S. Ullrich, Photoinduced Phenomena in Nucleic Acids I, Top. Curr. Chem., Springer, 2015, vol. 355.

8 M. Barbatti, A. C. Borin and S. Ullrich, Photoinduced Processes in Nucleic Acids. I, Top. Curr. Chem., Springer, 2015, vol. 355, pp. 1-32.

9 H. R. Hudock, B. G. Levine, A. L. Thompson, H. Satzger, D. Townsend, N. Gador, S. Ullrich, A. Stolow and T. J. Martínez, J. Phys. Chem., 2007, 111, 8500-8508.

10 S. Ullrich, T. Schultz, M. Z. Zgierski and A. Stolow, Phys. Chem. Chem. Phys., 2004, 6, 2796-2801.

11 C. Z. Bisgaard, H. Satzger, S. Ullrich and A. Stolow, ChemPhysChem, 2009, 10, 101-110.

12 Y. Harada, T. Suzuki, T. Ichimura and Y.-Z. Xu, J. Phys. Chem. B, 2007, 111, 5518-5524. 
13 Y. Harada, C. Okabe, T. Kobayashi, T. Suzuki, T. Ichimura, N. Nishi and Y.-Z. Xu, J. Phys. Chem. Lett., 2010, 1, 480-484.

14 C. Reichardt and C. E. Crespo-Hernández, J. Phys. Chem. Lett., 2010, 1, 2239-2243.

15 H. Kuramochi, T. Kobayashi, T. Suzuka and T. Ichimura, J. Phys. Chem. B, 2010, 114, 8782-8789.

16 C. Reichardt and C. E. Crespo-Hernández, Chem. Commun., 2010, 46, 5963-5965.

17 C. Reichardt, C. Guo and C. E. Crespo-Hernández, J. Phys. Chem. B, 2011, 115, 3263-3270.

18 M. Pollum, S. Jockusch and C. E. Crespo-Hernández, J. Am. Chem. Soc., 2014, 136, 17930-17933.

19 M. Pollum, S. Jockusch and C. E. Crespo-Hernández, Phys. Chem. Chem. Phys., 2015, 17, 27851-27861.

20 M. Pollum, L. Martínez-Fernández and C. E. CrespoHernández, in Photoinduced Phenomena in Nucleic Acids I, Top. Curr. Chem., ed. M. Barbatti, A. C. Borin and S. Ullrich, Springer, 2015, vol. 355, pp. 245-327.

21 S. Mai, M. Pollum, L. Martínez-Fernández, N. Dunn, P. Marquetand, I. Corral, C. E. Crespo-Hernández and L. González, Nat. Commun., 2016, 7, 13077.

22 M. Pollum, L. A. Ortiz-Rodríguez, S. Jockusch and C. E. Crespo-Hernández, Photochem. Photobiol., 2016, 92, 286-292.

23 B. Ashwood, S. Jockusch and C. E. Crespo-Hernández, Molecules, 2017, 22, 379.

24 L. Martínez-Fernández, G. Granucci, M. Pollum, C. E. Crespo-Hernández, M. Persico and I. Corral, Chem. - Eur. J., 2017, 23, 2619-2627.

25 P. O’Donovan, C. M. Perrett, X. Zhang, B. Montaner, Y.-Z. Xu, C. A. Harwood, J. M. McGregor, S. L. Walker, F. Hanaoka and P. Karran, Science, 2005, 309, 1871-1874.

26 X. Zhang, G. Jeffs, X. Ren, P. O'Donovan, B. Montaner, C. M. Perrett, P. Karran and Y.-Z. Xu, DNA Repair, 2007, 6, 344-354.

27 P. Karran and N. Attard, Nat. Rev. Cancer, 2008, 8, 24-36.

28 M. Pollum, L. Guan, S. Ahsanuddin, E. Baron, M. Lam and C. E. Crespo-Hernández, J. Invest. Dermatol., 2016, 136, S105.

29 O. Reelfs, P. Karran and A. R. Young, Photochem. Photobiol. Sci., 2012, 11, 148-154.

30 G. Trigiante and Y.-Z. Xu, in Photodynamic Therapy: Fundamentals, Applications and Health Outcomes, ed. A. G. Hugo, Nova Science, 2015.

31 M. Pollum and C. E. Crespo-Hernández, J. Chem. Phys., 2014, 140, 071101.

32 V. Vendrell-Criado, J. A. Sáez, V. Ljiaubet-Vallet, M. C. Cuquerella and M. A. Miranda, Photochem. Photobiol. Sci., 2013, 12, 1460-1465.

33 H. Yu, J. A. Sanchez-Rodriguez, M. Pollum, C. E. CrespoHernández, S. Mai, P. Marquetand, L. González and S. Ullrich, Phys. Chem. Chem. Phys., 2016, 18, 20168-20176.

34 G. Cui and W. Fang, J. Chem. Phys., 2013, 138, 044315.

35 J. P. Gobbo and A. C. Borin, Comput. Theor. Chem., 2014, 1040-1041, 195-201.

36 S. Mai, P. Marquetand and L. González, J. Phys. Chem. A, 2015, 119, 9524-9533.

37 S. Mai, P. Marquetand and L. González, J. Phys. Chem. Lett., 2016, 7, 1978-1983.
38 M. A. El-Sayed, J. Chem. Phys., 1963, 38, 2834-2838.

39 N. L. Evans and S. Ullrich, J. Phys. Chem. A, 2010, 114, 11225-11230.

40 H. Yu, N. L. Evans, A. S. Chatterley, G. M. Roberts, V. G. Stavros and S. Ullrich, J. Phys. Chem. A, 2014, 118, 9438-9444.

41 H. Yu, N. L. Evans, V. G. Stavros and S. Ullrich, Phys. Chem. Chem. Phys., 2012, 14, 6266-6272.

42 N. L. Evans, H. Yu, G. M. Roberts, V. G. Stavros and S. Ullrich, Phys. Chem. Chem. Phys., 2012, 14, 10401-10409.

43 T. J. Godfrey, H. Yu and S. Ullrich, J. Chem. Phys., 2014, 141, 044314.

44 A. Khvorostov, L. Lapinski, H. Rostkowska and M. J. Nowak, J. Phys. Chem. A, 2005, 109, 7700-7707.

45 J. H. Eland, Int. J. Mass Spectrom. Ion Phys., 1969, 2, 471-484. 46 C. Reichardt, R. A. Vogt and C. E. Crespo-Hernández, J. Chem. Phys., 2009, 131, 224518.

47 C. Reichardt, C. Wen, R. A. Vogt and C. E. CrespoHernández, Photochem. Photobiol. Sci., 2013, 12, 1341-1350.

48 J. Finley, P. Å. Malmqvist, B. O. Roos and L. Serrano-Andrés, Chem. Phys. Lett., 1998, 288, 299.

49 B. O. Roos, R. Lindh, P. Å. Malmqvist, V. Veryazov and P. O. Widmark, J. Phys. Chem. A, 2004, 108, 2851-2858.

50 M. J. Bearpark, M. A. Robb and H. B. Schlegel, Chem. Phys. Lett., 1994, 223, 269.

51 L. Serrano-Andrés, M. P. Fülscher and G. Karlström, Int. J. Quantum Chem., 1997, 65, 167-181.

52 F. Aquilante, J. Autschbach, R. K. Carlson, L. F. Chibotaru, M. G. Delcey, L. De Vico, I. Fdez. Galván, N. Ferré, L. M. Frutos, L. Gagliardi, M. Garavelli, A. Giussani, C. E. Hoyer, G. Li Manni, H. Lischka, D. Ma, P. Å. Malmqvist, T. Müller, A. Nenov, M. Olivucci, T. B. Pedersen, D. Peng, F. Plasser, B. Pritchard, M. Reiher, I. Rivalta, I. Schapiro, J. SegarraMartí, M. Stenrup, D. G. Truhlar, L. Ungur, A. Valentini, S. Vancoillie, V. Veryazov, V. P. Vysotskiy, O. Weingart, F. Zapata and R. Lindh, J. Comput. Chem., 2016, 37, 506-541.

53 C. Puzzarini, M. Biczysko, V. Barone, I. Peña, C. Cabezas and J. L. Alonso, Phys. Chem. Chem. Phys., 2013, 15, 16965-16975.

54 M. K. Shukla and J. Leszczynski, J. Phys. Chem. A, 2004, 108, 10367-10375.

55 A. R. Katritzky, M. Szafran and G. Pfister-Guillouzo, J. Chem. Soc., Perkin Trans. 2, 1990, 871-876.

56 M. Ruckenbauer, S. Mai, P. Marquetand and L. González, J. Chem. Phys., 2016, 144, 074303.

57 F. Laermer, T. Elsaesser and W. Kaiser, Chem. Phys. Lett., 1989, 156, 381.

58 When using a pump wavelength of $316 \mathrm{~nm}$, a higher concentration of 2-thiouracil was used in order to improve the signal to noise ratio because the ground-state molar absorptivity of 2thiouracil is much smaller at $316 \mathrm{~nm}$ than at 268 or $290 \mathrm{~nm}$. This is likely the reason for the observation of triplet self-quenching at $316 \mathrm{~nm}$, but not at the other two excitation wavelengths.

59 K. Taras-Goślińska, G. Burdziński and G. Wenska, J. Photochem. Photobiol., A, 2014, 275, 89.

60 S. Bai and M. Barbatti, Phys. Chem. Chem. Phys., 2017, 19, 12674-12682.

61 J. P. Zobel, J. J. Nogueira and L. González, Chem. Sci., 2017, 8, 1482-1499. 